\title{
THE FORMATION AND EVOLUTION OF COMPACT STARS IN BINARIES
}

\author{
Ronald E. Taam ${ }^{1}$ \\ RESUMEN
}

Se describen los procesos evolutivos estelares responsables de la formación de objetos compactos en sistemas binarios en interacción y su evolución. La fase de envolvente común juega un papel crucial en su formación y las pérdidas del momento angular relacionadas con el frenado magnético y/o pérdida de masa son importantes para su evolución. La aplicación de estos procesos proporciona el eslabón evolutivo entre las clases de sistemas binarios inter-actuantes.

\section{ABSTRACT}

The stellar evolutionary processes responsible for the formation of compact objects in interacting binary systems and their evolution are described. The common envelope phase plays a crucial role in their formation and angular momentum losses associated with magnetic braking and/or mass loss are important for their evolution. An application of these processes provides the evolutionary link between classes of interacting binary systems.

\section{Key Words: BINARIES: CLOSE - STARS: EVOLUTION}

\section{i. INTRODUCTION}

The recent Chandra observations of the X-ray point source population in the Galaxy and in external galaxies, in particular, have led to a resurgence of interest in the study of binary stars containing compact objects. An understanding of the nature of these sources depends critically upon our knowledge of the stellar evolutionary processes responsible for their formation and evolution. Generally, there is a consensus that many classes of interacting binary systems require a nonconserative form of evolution in which the loss of mass and orbital angular momentum play a fundamental role. Among systems which have undergone such an evolution are those with a white dwarf (WD), neutron star (NS), or black hole (BH) component.

The mechanisms by which significant mass and angular momentum can be removed from the binary system are thought to result from one or a combination of processes involving the binary interaction, continuous mass loss associated with stellar winds, or the nearly instantaneous mass ejection process associated with a supernova explosion. In this contribution we primarily focus on their influences in providing a framework for our understanding of the origin and evolution of these binary star systems.

\section{FORMATION OF COMPACT OBJECTS}

Binary interactions can play an important role in determining the ultimate state of a compact object

\footnotetext{
${ }^{1}$ Department of Physics and Astronomy, Northwestern University, Evanston, IL.
}

since their progenitors as components in binary star systems can lose a far greater fraction of their mass as a result of Roche lobe overflow as compared to their loss of mass via stellar winds as single stars. In the systems for which the two components are sufficiently close to each other such that gravitational tidal interactions are effective, the lower mass limits for the formation of compact objects are increased relative to their isolated counterparts. For example. stars less massive than $2.3 \mathrm{M}_{\odot}$ can evolve to $\mathrm{CO}$ WDs as a single star, but their evolution can lead to the formation of He WDs in sufficiently close interacting binary systems. Since the formation of a $W D$ compact object results from the loss of a stellar envelope, the mass transfer process can accelerate its removal, thereby truncating further growth of the core. For higher mass progenitors, the composition of the WD shifts to the heavier $\mathrm{CO}$ and $\mathrm{ONeMg}$ type. For cases in which the binary interaction leads to the exposure of a bare nondegenerate He core. its further evolution (for masses $\gtrsim 0.35 \mathrm{M}_{\ni}$ ) can lead to the formation of a hybrid WD with a thick helium-rich envelope overlying a $\mathrm{CO}$ core.

Neutron stars, on the other hand, are generally formed during a supernova explosion as a result of the core collapse of massive stars. The lower mass limit for their formation also depends on whether the star is single or in a binary. Specifically single stars $\gtrsim 8 \mathrm{M}_{\odot}$ can form NSs, whereas the lower mass iimit for NS formation in binaries may be as high as $12 \mathrm{M}_{\odot}$. For those supernova which are devoid of hydrogen-rich matter in their spectra, the progenitor 
must have undergone severe mass loss via a stellar wind (perhaps enhanced by tidal effects) or Roche lobe overflow. Binary interactions also make possible another channel for NS formation via the accretion induced collapse (AIC) of massive ONeMg WDs ( $\left.1.15 \mathrm{M}_{\odot}\right)$. Such WDs form from progenitor stars in the range of $\sim 6-12 \mathrm{M}_{\odot}$ in binary systems.

The fate of the accreting ONeMg WD critically depends upon its ability to accumulate mass, which may not be very efficient since matter can be ejected as a result of the activation of an unstable nuclear burning shell. Possible pathways which allow for the build up of the ONeMg WD to near the Chandrasekhar limit and to the AIC initiated by electon capture processes involve phases during which the rate of mass transfer is rapid. This can occur, for example, in CV-like systems in which a more massive main sequence-like donor transfers mass on a thermal timescale to its WD companion at orbital periods, $P$, of a few days (see Li \& van den Heuvel 1997; Ivanova \& Taam 2004) or for systems evolving on a nuclear burning timescale during the red giant (RG) stage ( $\mathrm{Li} \&$ van den Heuvel 1997). Of particular import in enhancing the viability of this channel was the recognition by Kato \& Hachisu (1994) that at high mass transfer rates $\left(\gtrsim 10^{-6} \mathrm{M}_{\odot} \mathrm{yr}^{-1}\right)$ an optically thick wind can be driven from the WD surface. Such a wind prevents the WD photosphere from expanding to RG dimensions and, hence, stabilizes the mass transfer process in the system. Although the efficiency of mass accumulation is reduced by a wind from the WD surface (Kato \& Hachisu 1999), it is not so inefficient to prevent the WD from increasing its mass to $1.39 \mathrm{M}_{\odot}$ at which point AIC occurs (Kondo \& Nomoto 1991).

The AIC phase for the formation of a NS can also occur for shorter period systems $(\lesssim 1$ hour) where a helium star or WD donor transfers matter onto an ONeMg WD (Belczynski \& Taam 2004). In this case the mass transferred is hydrogen poor and the off center ignition results of Kawai, Saio, \& Nomoto (1987) indicate that such WDs can evolve to AIC.

The core collapse of even more massive stars results in the formation of BHs. Direct collapse to a $\mathrm{BH}$ is thought to occur for stars $\gtrsim 40 \mathrm{M}_{\odot}$ (MacFadyen, Woosley, \& Heger 2001), whereas their formation is precipitated by the fall back of matter for stars in the mass range between about 25 and 40 $\mathrm{M} \ldots$. In an analogous process, a $\mathrm{BH}$ compact object can form in a binary system as a result of an AIC of a NS when its mass exceeds its maximum value. We note that the efficiency of the accretion process may be less than unity if a radiatively driven mass out- flow develops. Such outflows are expected in phases where either the mass transfer rate exceeds the Eddington rate (King \& Ritter 1999) or the mass flow within the disk surrounding the NS is temporarily increased above the Eddington limit as a result of a thermal/viscous instability during an X-ray transient outburst state (Taam, King, \& Ritter 2000). Estimates for the maximum mass of NSs above which $\mathrm{BH}$ formation may be possible via this pathway are in the range of $1.8-2.3 \mathrm{M}_{\odot}$ (Akmal, Pandharipande, \& Ravenhall 1998).

\section{MASS AND ANGULAR MOMENTUM LOSS}

It is generally accepted that the common envelope (CE) phase of evolution is the primary channel leading to the formation of compact objects in short period systems (Iben \& Livio 1993; Taam \& Sandquist 2000). In this phase, the two components of a primordial binary system are embedded within a differentially rotating CE. The failure to achieve corotation in the system, resulting from an unstable mass transfer phase or a tidal instability, leads to the inspiral of the two components. The successful ejection of the envelope necessitates that sufficient orbital energy is released to unbind the CE from the core of the more evolved component and its companion. That is, sufficient energy must be released from the orbit to unbind the CE from both cores. It should be noted that a condition based on an energetic argument is only a necessary condition, and it is not sufficient to guarantee the survival of a remnant binary system since the ejection timescale must be shorter than the inspiral timescale for the two cores. The latter condition can be satisfied for stars with a giant-like structure since the density gradients above the nuclear burning shells are sufficiently steep that the gravitational tidal interactions torque up little matter resulting in a significant deceleration of the inspiral process. Alternatively, for systems in which the mass of the companion and the $\mathrm{CE}$ are comparable, significant spin up can occur (Sandquist, Taam, \& Burkert 2000), leading to a concomittant reduction of the gravitational torque and, hence, to a deceleration of the orbital decay process.

After the formation of a compact object in a short period system, further angular momentum loss processes can play an important role in driving mass transfer in the system. Ever since the pioneering study by Verbunt \& Zwaan (1981), angular momentum losses associated with magnetic braking have been invoked to promote mass transfer in systems with low mass donors. For this process, as well as gravitational radiation, significant orbital angu- 
lar momentum can be lost from the system without necessarily requiring significant mass loss.

Recently, there have been several developments which indicate that magnetic braking, as applied to short period binary systems, is not as effective as has been hitherto assumed. We note that the angular momentum loss rates previously employed for examining the evolution of low mass binary systems are based on observations of slowly rotating $G$ type stars (Skumanich 1972). The angular momentum loss rate, $\dot{J}$, is proportional to $\Omega^{3}$, where $\Omega$ is the stellar angular velocity. The observations of rotational velocities of single stars in stellar clusters suggest that $\dot{J}$ at high rotation rates is reduced relative to an extrapolation based on low rotation rates; and an empirical prescription in the form $\dot{j} \times \Omega$ was proposed by Andronov, Pinsonneault, \& Sills (2003). An alternative approach was adopted by Ivanova \& Taam (2003), who estimated $\dot{J}$ from a two component magnetically coupled stellar wind model (Mestel \& Spruit 1987) prescribed to reproduce the non saturated and saturated coronal X-ray emission from main sequence stars (Pizzolato et al. 2003). The angular velocity dependence of the X-ray luminosity was shown by Ivanova \& Taam (2003) to be sensitive to the variation of the coronal density, $\rho$, with rotation rate. For $\rho \propto \Omega^{0.6}$, the non saturated behavior found by Pizzolato et al. (2003) $\left(L_{x} \propto \Omega^{2}\right)$ as well as the saturated behavior was reproduced. The resulting angular momentum loss rate in this model smoothly varied from $\dot{J} \propto \Omega^{3}$ at low rotation rates to $\dot{J} \propto \Omega^{1.3}$ at high rotation rates. An application of this prescription to the evolution of CVs (Ivanova $\&$ Taam 2003) led to mass transfer rates which are in accordance with observations (Patterson 1984).

Angular momentum loss associated with mass loss can also be important in stable mass transferring systems. For example, mass loss associated with a stellar wind carries the specific orbital angular momentum of the binary component. A more efficient orbital angular momentum loss process is possible if matter exists in the form of a gaseous disk surrounding the system. Such a disk may result from the gravitational focusing of the slowly moving ejected matter into the equatorial plane (Spruit \& Taam 1981) from either of the stellar components or from the accretion disk surrounding the accretor. Here, gravitational tidal torques lead to the transfer of orbital angular momentum to the disk, causing it so spread outwards. This transfer of angular momentum can feedback on the binary promoting the mass transfer itself. Such studies have been applied to CVs (Taam, Sandquist, \& Dubus 2003), showing that such disks can be effective at promoting mass transfer rates as high as $10^{-8} \mathrm{M}, \mathrm{yr}^{-1}$ even for very low fractional mass input rates $\left(\sim 10^{-4}\right)$ into the disk.

\section{EVOLUTIONARY LINKS TO CLASSES OF SYSTEMS}

As described previously the CE phase is a critical link in facilitating the transformation of long period systems into short period compact binary systems. The numerical results of $3 \mathrm{D}$ hydrodynamical simulations reveal that the successful ejection of the $\mathrm{CE}$ is more favorable for progenitors which have evolved beyond the Hertzprung gap to the RG and asymptotic branch phase. Survival as a remnant binary for low and intermediate mass binaries is likely for He cores $\gtrsim 0.2 \mathrm{M}_{6}$, on the $\mathrm{RG}$ branch and $\mathrm{CO}$ cores $\gtrsim 0.6 \mathrm{M}_{\odot}$ on the asymptotic giant branch. In all cases there is significant orbital shrinkage (more than a factor of 50) resulting in the reduction of the orbital period from a year to $\lesssim$ a day (see Taam \& Sandquist 2000).

As these systems are detached in the post $\mathrm{CE}$ phase, either angular momentum losses and/or nuclear evolution of the non compact component of the binary is necessary to force the systems to the mass transfer stage. Their subsequent evolution can give rise to the formation of CVs with evolved or unevolved secondaries (i.e., AM CVn systems, double WDs: see Podsiadlowski, Han, \& Rappaport 2003; Ivanova \& Taam 2004).

For binaries with intermediate or massive progenitors, the CE can be successfully ejected for stars beyond the core He burning stage. The subsequent evolution of the core remnant can lead to the formation of a NS during the core collapse stage for He cores $\gtrsim 2.8 \mathrm{M}_{\odot}$. Provided that the system remains bound after the supernova stage, the system can evolve to the low mass X-ray binary (LMXB) phase directly from the common envelope phase or from an intermediate mass X-ray binary (IMXB) phase (Podsiadlowski, Rappaport, \& Pfahl 2002).

Additional pathways to the LMXB and IMXB phase involve the electron capture AIC process of ONeMg WDs. One such channel involves the thermal mass transfer phase of CV's with main sequencelike donors $\gtrsim 1.8 \mathrm{M}$, , but less massive than a critical value given by the mass ratio $(\sim 3)$ above which a delayed dynamical instability occurs (Iranova of Taam 2004). Another channel involves either $\mathrm{He}$ star or WD donors in systems with $P \lesssim 1$ hour (Belczrnski \& Taam 2004). The former chamnel contributes to the IMIXB or LMXX phase. whereas the latter channel only leads to the formation of LMKBs at ultrashort orbital periods. 
The fate of the LMXBs likely leads to the formation of binary millisecond pulsars (BMSPs). Interestingly, the orbital period distribution of such systems reveals evidence of a gap between 23 and 56 days (Taam, King, \& Ritter 2000). The division of these systems into those characterized by $P \gtrsim 60$ days and those characterized by $P \lesssim 30$ days is natural and can be understood from the fact that for comparable initial orbital periods ( $\gtrsim$ a few days) low mass Case $B$ evolution leads to long final orbital periods and early massive Case B evolution lead to short final orbital periods. A population synthesis analysis carried out by Willems \& Kolb (2002) confirms this interpretation, but they point out the difficulty of producing BMSPs in the orbital period range between $\sim 1-3$ days. A possible resolution to this lack of agreement may lie with the alternative pathway involving a thermal mass transfer phase in a $\mathrm{CV}$ where the NS is formed via an AIC. In this evolutionary channel, the timescale of evolution may be sufficiently long after the mass ratio is reversed to allow significant growth of the He core. Intermediate mass stars $(\sim 2 \mathrm{M}$.$) are ideal progenitors for these$ systems since they favor the formation of both an AIC of an ONeMg WD (Ivanova \& Taam 2004) and a remnant core of the order of a few tenths of a solar mass. Although lower-mass main sequence-like stars could also produce remnant WD masses as high as $0.2 \mathrm{M}_{\odot}$, such systems are not viable progenitors for these BMSPs since the orbital period of the system would significantly increase beyond 3 days.

\section{FUTURE WORK}

Due to the uncertainties in the fundamental processes governing the nonconservative nature of the evolution of binaries with compact objects, there are many areas where further work would be fruitful. The most important phase which requires a quantitative description is the outcome of the common envelope phase. High resolution calculations are currently being carried out in order to determine the efficiency of ejection of this phase for a wide range of orbital periods (i.e., evolutionary states) and component masses. Such studies will be useful for delineating the parameter regimes (orbital period, and mass of the two components) where a post spiral in system survives and where the binary system merges.

For processes invoking significant angular momentum loss without significant mass loss, the extension of the study of magnetic activity and rotation from main sequence stars to evolved stars is desirable in understanding the evolution of systerns dominated by angular momentum losses.
The existence of radiatively driven optically thick wind solutions from the WD surface (Kato \& Hachisu 1994) has expanded the possible evolutionary channels for the formation of near Chandrasekhar Type Ia supernova models and NSs via the AIC process. The build up of the WD depends critically on the accumulation efficiency of matter in the presence of unstable He burning shells. More extensive studies of this phase over a wide range of WD masses are urged.

Finally, the incorporation of these inputs into evolutionary sequences for population synthesis studies are essential for obtaining an understanding of the formation rates of these systems.

This work has been supported by in part by NSF under grant AST-0200876.

\section{REFERENCES}

Akmal, A., Pandharipande, V. R., \& Ravenhall, D. G. 1998, Phys. Rev. C, 58, 180

Andronov, N., Pinsonneault, M., \& Sills, A. 2003, ApJ, 582,358

Belczynski, K., \& Taam, R. E. 2004, ApJ, 603, in press Iben, I. Jr., \& Livio, M. 1993, PASP, 105, 1373

Ivanova, N., \& Taam, R. E. 2003, ApJ, 599, 516

Ivanova, N., \& Taam, R. E. 2004, ApJ, 601, in press

Kato, M., \& Hachisu, I. 1994, ApJ, 437, 832

Kato, M., \& Hachisu, I. 1999, ApJ, 513, L41

Kawai, Y., Saio, H., \& Nomoto, K. 1987, ApJ, 315, 229

King, A. R., \& Ritter, H. 1999, MNRAS, 309, 253

Kondo, Y., \& Nomoto, K. 1991, ApJ, 367, L19

Li, X. D., \& van den Heuvel, E. P. J. 1997, A\&A, 322, L9

MacFadyen, A. I., Woosley, S. E., \& Heger, A. 2001, ApJ, 550,410

Mestel, L., \& Spruit, H. C. 1987, MNRAS, 226, 57

Patterson, J. 1984, ApJS, 54, 443

Pizzolato, N., Maggio, A., Micela, G., Sciortino, S., \& Ventura, P. 2003, A\&A, 397, 147

Podsiadlowski, Ph., Han, Z, \& Rappaport, S. 2003, MNRAS, 340, 1214

Podsiadlowski, Ph., Rappaport, S., \& Pfahl, E. D. 2002, ApJ, 565, 1107

Sandquist, E. L., Taam, R. E., \& Burkert, A. 2000, ApJ, 533,984

Skumanich, A. 1972, ApJ, 171, 565

Spruit, H. C. \& Taam, R. E. 2001, ApJ, 548, 900

Taam, R. E., King, A. R., \& Ritter H. 2000, ApJ, 541, 329

Taam, R. E., \& Sandquist, E. L. 2000, ARA\&A, 38, 113

Taam, R. E., Sandquist, E. L., \& Dubus, G. 2003, ApJ, 592,1124

Verbunt, F., \& Zwaan, C. 1981, A\&A, 100, L7

Willems, B., \& Kolb, U. 2002, MNRAS, 337, 1004 